\title{
ICMAT 2011 conference held in Singapore
}

www.mrs.org.sg/icmat2011

T he sixth International Conference on Materials for Advanced Technologies (ICMAT-2011), organized by the Materials Research Society of Singapore (MRS-S), was held on June 26 to July 1, 2011 in Singapore. The program covered various aspects of materials science, engineering, and technology, including the science and technology of graphene; topological insulators; nanostructures of various materials; metamaterials; photovoltaics, including dyesensitized solar cells; and lithium-ion battery materials for electric vehicles (EVs). A few of the research highlights follow.

Nobel laureate Andre Geim (University of Manchester, UK), in a plenary talk, enumerated the unique physical and chemical properties of graphene (a single layer of carbon atoms) and why scientists and engineers throughout the world are excited about exploiting it for potential applications. Geim said that during the last two years the quality of synthetic graphene has improved greatly. Eventually, manufacturers will have the ability to make graphene in ton quantities and kilometer lengths. A transparent, flexible, rectangular sheet of graphene with a diagonal of 30 inches has already been made, he said. Low-temperature chargecarrier mobilities of a few million $\mathrm{cm}^{2} /$ Vs have been demonstrated. Geim said that when exposed to fluorine, graphene becomes a wide-bandgap semiconductor and the fluorographene also acts as "twodimensional Teflon."

Kicking off the symposium on Advanced Materials for Energy Storage Systems (symposium N), Keynote Speaker Gebrand Ceder of the Massachusetts Institute of Technology, USA (MIT) showed recent results on the Liion battery cathode material $\mathrm{LiFePO}_{4}$. His group studied the system with a combination of first-principles calculations and molecular dynamics simulations and calculated the mixing energy as a function of Li concentration. They find that $30 \mathrm{meV}$ of overpotential is required to access the solid solution, and furthermore that neighboring nanoparticles of $\mathrm{LiFePO}_{4}$ go through the solid solution sequentially during cycling, one giving up all of its Li before its neighbor begins to de-lithify. He said that the absence of nucleation in this model is much more compatible with the observed high transformation rates and posed the question of whether this model can be extended to other fast two-phase systems, like $\mathrm{LiMnPO}_{4}$ and $\mathrm{Li}_{4} \mathrm{Ti}_{5} \mathrm{O}_{12}$. Furthermore, he showed a sampling of data from the Materials Genome Project, a database project he founded in order to help researchers data-mine scientific trends in materials properties. The project has thus far generated and compiled data for over 15,000 compounds, focusing on potential electrode materials for $\mathrm{Li}$ ion batteries. To demonstrate the power of the approach, Ceder discussed some of the results comparing the stability of a range of oxides and phosphates. Though conventional wisdom holds that phosphates are much safer than oxides, his data mining found that oxides are only moderately less stable than their phosphate counterparts at a given voltage.

In symposium $\mathrm{S}$ on Metamaterials, Nikolay Zheludev (University of Southampton, UK) reported that his group's goal is the development of nonlinear, switchable metamaterials for all-optical data processing, storage, and telecom switching. He defined the ultimate non- linear medium as one in which "all atoms within the light absorption length absorb a photon." Zheludev described four possible paths toward the goal: (1) hybrid metamaterials consisting of a nonlinear medium plus a plasmonic metamaterial; (2) a phase-change material plus a plasmonic metamaterial; (3) a reconfigurable metamaterial that can change size mechanically; and (4) a flux quantization metamaterial. Various materials his group has considered and investigated are carbon nanotubes (CNTs) and graphene coupled with a metamaterial (gold) or with nonlinear metamaterials and superconducting metamaterials (e.g., yttrium barium copper oxide and $\mathrm{Nb}$ ). The latter two are interesting because of their extraordinary transmission capabilities and

MRS

Fano resonances (a type of resonant scattering phenomena that gives rise to an asymmetric line-shape), Zheludev said.

Masafumi Yamaguchi (Toyota Technological Institute, Japan), in symposium O (Photovoltaic Materials and Devices), presented Japan's roadmap, up to 2025, for photovoltaic (PV) cells. Their mid- to long-term plan slates nearly 30 billion yen ( $\sim$ USD $\$ 385$ million) to reach a target of 14 yen ( $\sim$ USD\$0.18) per kilowatthour by 2020 , with a module production cost of 75 yen ( USD $\$ 1)$ per watt. Target module conversion efficiencies for that period range from $20 \%$ for wafer-based Si technologies to $10 \%$ for dye-sensitized solar cells. Yamaguchi particularly highlighted the push in multijunction cells, as demonstrated by his research group, which seeks to aim for efficiencies of $45 \%$ for a cell and $35 \%$ for a module.

In the same symposium, Andreas Bett (Fraunhofer Institute for Solar Energy Systems, Germany) made the case for

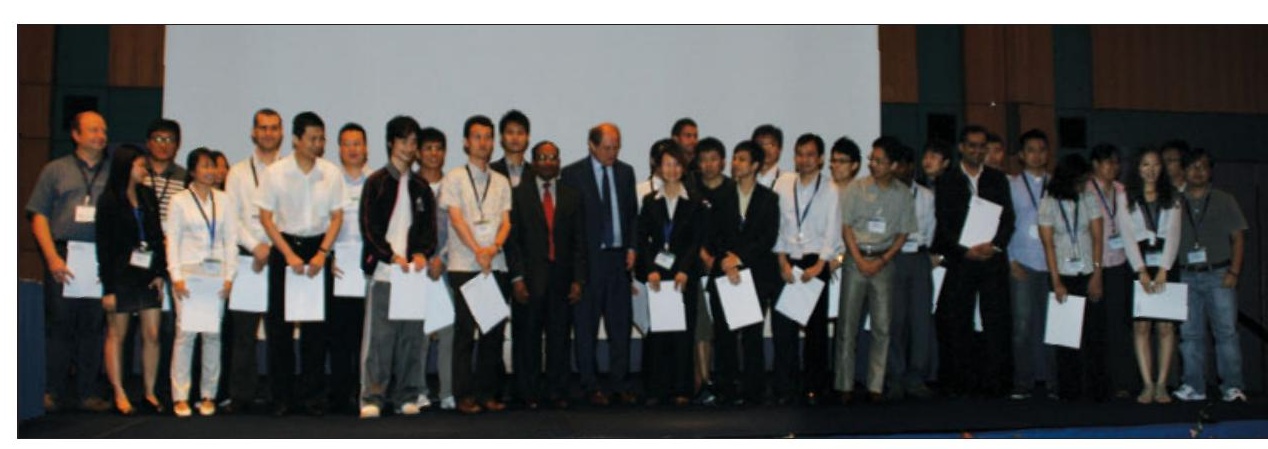


Singapore) discussed the design, development, and testing of polymeric materials for biodegradable cardiovascular implants. His group's goal is to replace the presently used metallic stents with a polymeric stent that self-expands at body temperature, maintains its structural integrity for three to six months, fully degrades after nine months, and releases multiple drugs at controlled rates over the lifetime of the stent. They experimented and succeeded with doublelayered hollow tubes, with the interior layer made of the polymer poly-(L-lactic acid) (PLLA) and the outer layer of poly-(lactic-glycolic acid) (PLGA), both biodegradable polymers. By varying the thickness of the layers, the rate of degradation in the body could be controlled. Boey said that their polymers have the benefit of incorporating drugs into their structures, which is an improvement over metal stents that can only support drugs on their surfaces. This allows an infusion of a higher concentration of drugs into a layer, and to vary the drug type from layer to layer, if desired.

Boey reported great success with this approach in tests carried out on animals. The polymer stents self-expand in 3-10 min to open the clogged blood vessel; they degrade layer-by-layer at rates controlled by the thickness and composition of the layers; they deliver high concentrations of drugs over a long period of time; they encourage overgrowth of a protective epithelial layer; and they biodegrade inside this epithelial pocket, so that the degradation by-products do not enter the bloodstream, where they might cause a stroke. Cross sections of removed blood vessels revealed an open artery and good blood flow in the region of the degraded stent after 60 days. Boey said that much more testing needs be performed before these biodegradable polymer stents might be ready for use in humans.

In symposium F (Toxicology of Engineered Nanomaterials), Suresh Balasubramanian of the National University of Singapore (NUS), reported results of studies on the effects of nanoparticles of gold, with diameters of $20 \mathrm{~nm}$ and 7 $\mathrm{nm}$, introduced into rats through inhalation and injection. Their group found that inhaled particles tend to accumulate primarily in the lungs and olfactory bulb (nasal pathway), although some were transported through the bloodstream to the aorta, spleen, and kidneys, for example. Injected particles tended to follow the bloodstream and deposit in organs across the body, although some made it back to the lungs. Surprisingly, Balasubramanian said, very few nanoparticles were found in the urine of the animals, which means that they are not passing through the animal at all. Such studies are of relevance, since there is growing concern over the toxicity of handling and use of nanoparticles to humans and the environment. It was pointed out that detailed studies of various systems of nanoparticles are urgently needed.

Other plenary talks of ICMAT 2011 were delivered by Nobel laureates Albert Fert (Unité Mixte de Physique CNRS/ Thales and the Univ. Paris-Sud, France), Ada Yonath (Weizmann Institute, Israel), and Klaus von Klitzing (Max-PlanckInstitut für Festkörperforschung, Germany), as well as Joachim Luther (Solar Energy Renewable Initiative of Singapore and NUS), Susumu Kitagawa (Kyoto University, Japan), Charles Lieber (Harvard University, USA), and Jean M.J. Frechet (King Abdullah University of Science and Technology, Saudi Arabia). Fert and Yonath also delivered Public Lectures at the NUS Cultural Center, which were open to the conference attendees as well as the general public, high school and junior college students. Three "Theme lectures" were given by Jonathan Adams (Thomson-Reuters, UK), Qi-Kun Xue (Tsinghua University, China), and D.D. Sarma (Indian Institute of Science, Bangalore, India).

The conference was inaugurated by the Minister for Trade and Industry, Lim Hng Kiang, Government of Singapore. Tan Chorh Chuan, President of NUS, and B.V.R. Chowdari, Organizing Chair of the ICMAT 2011 Conference, also from the NUS, welcomed the gathering on the first day of the weeklong conference. The Ambassador of France and the Indian High Commissioner also addressed the conference.

More reports can be found online at www.mrs.org/meeting-scene/.
T he Modern Practice in Stress and Vibration Analysis Conference will be held in Glasgow, UK, on August 2931, 2012. The conference will present international research in the fields of vibration analysis and stress analysis and technical areas where they intersect.

Among the confirmed speakers are J.R. Barber (University of Michigan, USA) who will present a talk on "Frictional systems under periodic loads"; W.
Lacarbonara (La Sapienza University, Rome, Italy), "Nonlinear dynamics enabled systems design and control"; $F$. Pierron (Arts et Métiers, Paris Tech, France), "A novel photomechanical approach to dynamic testing of materials"; W. Ostachowicz (Polish Academy of Sciences, Gdansk, Poland), "Structural health monitoring by means of elastic wave propagation"; and J. Warminski (Lublin University of Technology, Po- land), "Nonlinear phenomena in mechanical systems dynamics."

The abstract deadline is March 14, 2012.

The conference is organized by the Institute of Physics Applied Mechanics Group and co-sponsored by NAFEMS. It is endorsed by the Materials Research Society.

For further information, visit http:// mpsva2012.iopconfs.org. 